\title{
Identification of potential diagnostic biomarkers for pneumonia caused by adenovirus infection in children by screening serum exosomal microRNAs
}

\author{
FENG HUANG $^{1 *}, \mathrm{JUN} \mathrm{BAI}^{2 *}, \mathrm{JUNSONG} \mathrm{ZHANG}^{3 *}$, DIYUAN YANG ${ }^{1}$, \\ HUIFENG FAN $^{1}$, LI HUANG ${ }^{1}$, TINGTING SHI ${ }^{1}$ and GEN LU ${ }^{1}$ \\ ${ }^{1}$ Department of Respiratory Medicine, Guangzhou Women and Children's Medical Center, Guangzhou Medical University, \\ Guangzhou, Guangdong 510120; ${ }^{2}$ Department of Pediatrics, Hospital Foshan, Guangzhou, Guangdong 528000; \\ ${ }^{3}$ Institute of Human Virology, Zhongshan School of Medicine, Sun Yat-sen University, Guangzhou, \\ Guangdong 510080, P.R. China
}

Received September 2, 2018; Accepted February 7, 2019

DOI: $10.3892 / \mathrm{mmr} .2019 .10107$

\begin{abstract}
Human adenovirus (HAdV) infection causes serious pneumonia in children, leading to significant morbidity and mortality rates. However, diagnostic biomarkers for HAdV-associated pneumonia are unavailable. Serum microRNAs (miRNAs/miRs) have been recently reported as diagnostic biomarkers for several diseases. The present study performed microRNA sequencing to identify potential biomarkers among serum exosomal miRNAs, with the aim of identifying candidate biomarkers for the diagnosis of pneumonia in adenovirus-infected children. To validate the biomarker candidates, reverse transcription-quantitative polymerase chain reaction (RT-qPCR) was performed to determine the relative expression levels of miRNAs. As there is no endogenous reference RNA for serum miRNAs, pairwise analysis of RT-qPCR was used in the present study to narrow down the number of biomarker candidates among all the serum exosomal miRNAs to a set of four miRNAs. As a result, the identified miRNAs (namely, miR-450a-5p-miR-103a-3p and miR-103b-5p-miR-98-5p) from 59 samples were considered as potential diagnostic biomarkers in adenovirus-infected children. The results indicated that this four miRNA set could distinguish adenovirus-infected patients from healthy controls. In conclusion, the four exosomal miRNAs identified in the present study could be considered as candidate
\end{abstract}

Correspondence to: Dr Gen Lu, Department of Respiratory Medicine, Guangzhou Women and Children's Medical Center, Guangzhou Medical University, 9 North Huaqiang, Guangzhou, Guangdong 510120, P.R. China

E-mail: lugen5663330@sina.com

${ }^{*}$ Contributed equally

Key words: human adenoviruses, patients with pneumonia, serum microRNAs, diagnostic biomarkers diagnostic biomarkers for pneumonia in adenovirus-infected children.

\section{Introduction}

Pneumonia causes $\sim 1.3$ million mortalities in children each year $(1,2)$. Human adenovirus (HAdV) infection is one of the main causes of community-acquired pneumonia in children and young adults $(3,4)$. Adenoviruses are DNA viruses that typically cause infections in the upper or lower respiratory and gastrointestinal tracts, and the conjunctiva (5-7). Fatality rates for untreated severe HAdV-associated pneumonia may be $>50 \%$ each year (8). The traditional diagnosis of HAdV infection is based on evidence of positive, multiplex HAdV polymerase chain reaction (PCR) products from respiratory tract samples, including sputum or bronchoalveolar lavage (BAL) fluid (3). However, this technique has certain limitations, and is unable to provide an early and effective response to adenovirus infections of the lower respiratory tract. Therefore, it is necessary to identify novel methods for the diagnosis of HAdV pneumonia.

MicroRNAs (miRNAs or miRs) are single-stranded, 19-24-nucleotide (nt)-long RNA molecules that regulate gene expression and function at the post-transcriptional level (9). Serum miRNAs have been reported to be stable; certain miRNAs have been implicated in cancer pathogenesis, including breast and colorectal cancer (10-12). The use of serum/plasma miRNAs as novel, non-invasive biomarkers for the diagnosis of several diseases has been explored in previous studies $(13,14)$.

In the present study, to screen potential biomarkers for the diagnosis of HAdV pneumonia, exosomal miRNA profiles extracted from the serum of HAdV-infected or healthy children were compared. Exosomal miRNA expression was investigated as using the exosomal fraction increases the sensitivity of miRNA detection (15). Pairs of miRNAs were selected, which could be considered as candidate diagnostic biomarkers. These selected miRNA pairs could distinguish HAdV-infected children from uninfected children, indicating 
that such miRNAs may be used for the diagnosis of HAdV pneumonia in children.

\section{Materials and methods}

Preparation of serum samples. The blood samples from 29 healthy children and $30 \mathrm{HAdV}$ patients used in the present study were obtained from Guangzhou Women and Children's Medical Center (Guangzhou, China) between January 2015 and January 2019. The ages of all patients and healthy volunteers, including 33 males and 26 females, ranged from 1 to 13 years. HAdV pneumonia was diagnosed using the following criteria: i) Lower respiratory and/or systemic symptoms of infection; ii) computed tomography (CT) scan or lung infiltration on chest radiography; and iii) positive results for HAdV immunoglobulin M (IgM) antibody in serum and/or HAdV DNA by PCR in throat swabs and/or BAL fluid. We also excluded the samples from mixed infection patients who were infected with HAdV together with other microorganisms. Serum samples were prepared by centrifugation at $1,000 \mathrm{x} \mathrm{g}$ for $10 \mathrm{~min}$. The supernatants were collected and stored in aliquots at $-80^{\circ} \mathrm{C}$. Study approval was granted by the Ethics Committee at Guangzhou Women and Children's Medical Center (approval no. 2014121815), and all the parents or legal guardians of the patients signed written informed consent forms and agreed to its content.

Extraction of serum exosomes. Serum from all participants was collected for exosome isolation using the ExoQuick Exosome Precipitation kit (System Biosciences, LLC, Palo Alto, CA, USA) according to the manufacturer's protocol (16).

\section{Exosome characterization}

Transmission electron microscopy (TEM). Exosome suspensions in PBS $(100 \mu \mathrm{l})$ were added to a copper mesh placed on a clean wax plate. After $4 \mathrm{~min}$, the copper mesh was removed and placed in $2 \%$ phosphotungstic acid for $5 \mathrm{~min}$, while the mesh was dried on filter paper. The morphology of the exosomes was examined using a JEM-1230 transmission electron microscope (JEOL Ltd., Tokyo, Japan).

Western blot analysis. The exosome pellet was dissolved in radioimmunoprecipitation assay lysis buffer (Cell Signaling Technology, Inc., Danvers, MA, USA) for $30 \mathrm{~min}$ at $4^{\circ} \mathrm{C}$, and the protein concentration was determined using a Bradford protein assay kit (Bio-Rad Laboratories, Inc., Hercules, CA, USA) $(17,18)$. The proteins $(20 \mu \mathrm{g} /$ lane) were separated via $15 \%$ SDS-PAGE and then transferred to polyvinylidene difluoride membranes. Membranes were blocked in 5\% non-fat dried milk for $1 \mathrm{~h}$, followed by incubation for $1 \mathrm{~h}$ with anti-CD9 (1:1,000; cat. no. 13174, Cell Signaling Technology, Inc.) and anti-heat shock protein $90 \alpha$ (HSP90 $\alpha ; 1: 1,000$; cat. no. 8165, Cell Signaling Technology, Inc.) primary antibodies, and subsequent incubation for $1 \mathrm{~h}$ with the secondary antibodies (horseradish peroxidase-conjugated anti-rabbit immunoglobulin G; 1:1,000; cat. no. 7074, Cell Signaling Technology, Inc.). The bands were visualized using the SuperSignal chemiluminescence system (Thermo Fisher Scientific, Inc., Waltham, MA, USA). All steps were performed at room temperature.
RNA extraction from exosomes. RNA was extracted from the exosome pellets using TRIzol ${ }^{\circledR}$ (Thermo Fisher Scientific, Inc.) according to the manufacturer's protocol (17).

miRNA sequencing (miRNA-seq) and data analysis. Small RNA libraries were generated using NEBNext ${ }^{\circledR}$ Multiplex Small RNA Library Prep Set for Illumina ${ }^{\circledR}$ (New England BioLabs, Inc., Ipswich, MA, USA); all subsequent steps were conducted according to the manufacturer's protocol. Briefly, the multiplex 3 ' small RNA (SR) adapters, which the reverse transcription (RT) primers were hybridized to, were ligated to the miRNA. The RT step was performed upon ligation of the multiplex 5' SR adapter, and 15 cycles of PCR were performed to enrich those DNA fragments that had adapter molecules on both ends. PCR was performed as follows: $30 \mathrm{sec}$ at $94^{\circ} \mathrm{C}$, then $15 \mathrm{sec}$ at $94^{\circ} \mathrm{C}, 30 \mathrm{sec}$ at $62^{\circ} \mathrm{C}$, and $15 \mathrm{sec}$ at $70^{\circ} \mathrm{C}$ for 15 cycles. The library fragments were size-selected by $6 \%$ PAGE extraction. The purified DNAs of $\sim 140$ nt constituted the miRNA library. The libraries were quantified with using a Qubit 3.0 fluorometer (Invitrogen; Thermo Fisher Scientific, Inc.) and validated with an Agilent 2100 Bioanalyzer (Agilent Technologies, Inc., Santa Clara, CA, USA), prior to being sequenced on an Illumina HiSeq 2500 Sequencing System (Illumina, Inc., San Diego, CA, USA) for 50 cycles.

The adapter sequences were removed from all reads. Reads with Phred quality scores were $<10$ were truncated at their first nucleotides. Reads $<17 \mathrm{nt}$ were discarded. The remaining reads were mapped to a human miRNA reference sequence in the miRBase database (http://www.mirbase.org/) using the FANSe2 algorithm with parameters-L60-E2-U1-S10 (FANSe v2.0; http://bioinformatics.jnu.edu.cn/software/fanse2/). miRNA expression was quantified as reads per million. The differentially expressed miRNAs were detected using the edgeR package version 3.8 (R, https://bioconductor. org/packages/release/bioc/html/edgeR.html). miRNAs with $>2$ or $<0.5$-fold change, and $\mathrm{P}<0.01$ (no correction for Type I error was applied) were considered as differentially expressed miRNAs $(19,20)$.

RT-quantitative PCR (RT-qPCR). RNA was extracted from exosome pellets as aforementioned and the isolated RNAs were reverse transcribed using a SuperScript ${ }^{\mathrm{TM}}$ First-Strand Synthesis System for RT-PCR kit (cat. no. 11904018, Invitrogen; Thermo Fisher Scientific, Inc.) according to the manufacturer's protocol. qPCR was performed using the Power SYBR Green PCR Master Mix (Applied Biosystems; Thermo Fisher Scientific, Inc.) (17). Each reaction was performed in a 20- $\mu 1$ volume system including $5 \mu \mathrm{l}$ cDNA, $10 \mu 1$ Power SYBR Green PCR Master Mix, $0.5 \mu \mathrm{l}$ each primer and $4.0 \mu \mathrm{l}$ double distilled water. PCR was performed as follows: 5 min at $94^{\circ} \mathrm{C}$, then $20 \mathrm{sec}$ at $94^{\circ} \mathrm{C}$ and $30 \mathrm{sec}$ at $60^{\circ} \mathrm{C}$ for 39 cycles. The relative expression of miRNAs normalized to other miRNAs was calculated using the $2^{-\Delta \Delta \mathrm{Cq}}$ method $(15,21)$. All RT-qPCR primers used in the study are listed in Tables I-III.

Statistical analysis. Data was analyzed using SPSS software version 18.0 (SPSS, Inc., Chicago, IL, USA). Paired t-tests and two-way analyses of variance were performed to analyze the relative expression of exosomal miRNAs. $\mathrm{P}<0.01$ was considered to indicate a statistically significant difference. 
Table I. Nucleotide sequence of miRs.

\begin{tabular}{llc}
\hline miR & \multicolumn{1}{c}{ Sequence $^{\text {a }}\left(5^{\prime}-3^{\prime}\right)$} & Accession number $^{\mathrm{b}}$ \\
\hline hsa-miR-152-3p & UCAGUGCAUGACAGAACUUGG & MIMAT0000438 \\
hsa-miR-103a-3p & AGCAGCAUUGUACAGGGCUAUGA & MIMAT0000101 \\
hsa-miR-185-5p & UGGAGAGAAAGGCAGUUCCUGA & MIMAT0000455 \\
hsa-miR-98-5p & UGAGGUAGUAAGUUGUAUUGUU & MIMAT0000096 \\
hsa-let-7f-5p & UGAGGUAGUAGAUUGUAUAGUU & MIMAT0000067 \\
hsa-miR-206-5p & UGGAAUGUAAGGAAGUGUGUGG & MI0000490 \\
hsa-miR-450a-5p & UUUUGCGAUGUGUUCCUAAUAU & MIMAT0001545 \\
hsa-miR-145-5p & GUCCAGUUUUCCCAGGAAUCCCU & MIMAT0000437 \\
hsa-miR-103b-5p & UCAUAGCCCUGUACAAUGCUGCU & MIMAT0007402
\end{tabular}

${ }^{a}$ The sequences were downloaded from mirbase (http://www.mirbase.org/). ${ }^{\mathrm{b}}$ Accession numbers from miRBase. miR, microRNA.

Table II. Specific reverse transcription primer sequences used for nine miRs.

$\operatorname{miR}$

Reverse transcription primer sequence $\left(5^{\prime}-3^{\prime}\right)$

hsa-miR-152-3p

hsa-miR-103a-3p

hsa-miR-185-5p

hsa-miR-98-5p

hsa-let-7f-5p

hsa-miR-206-5p

hsa-miR-450a-5p

hsa-miR-145-5p

hsa-miR-103b-5p GTCGTATCCAGTGCAGGGTCCGAGGTATTCGCACTGGATACGACACCAAGTT
GTCGTATCCAGTGCAGGGTCCGAGGTATTCGCACTGGATACGACATCATAGC
GTCGTATCCAGTGCAGGGTCCGAGGTATTCGCACTGGATACGACATCAGGAA
GTCGTATCCAGTGCAGGGTCCGAGGTATTCGCACTGGATACGACAAACAATA
GTCGTATCCAGTGCAGGGTCCGAGGTATTCGCACTGGATACGACAAACTATA
GTCGTATCCAGTGCAGGGTCCGAGGTATTCGCACTGGATACGACACCACACA
GTCGTATCCAGTGCAGGGTCCGAGGTATTCGCACTGGATACGACAATATTAG
GTCGTATCCAGTGCAGGGTCCGAGGTATTCGCACTGGATACGACAAGGGATT
GTCGTATCCAGTGCAGGGTCCGAGGTATTCGCACTGGATACGACAAGCAGCA

miR, microRNA.

\section{Results}

Characteristics of patients with HAdV pneumonia. All patients enrolled in the present study were diagnosed with pneumonia based on clinical symptoms and lobar infiltrates on chest radiography. The main clinical symptoms were fever and cough, while two patients exhibited wheezing and tachypnea. All patients were HAdV-positive, with results from the HAdV IgM antibody test using serum and/or HAdV DNA PCR analysis using throat swabs and/or BAL fluid. The clinical characteristics of the cases are summarized in Table IV. Abnormal chest radiographs were also observed in all cases. Chest scans revealed diffuse infiltration of the lungs. Representative high-resolution CT scans of the chest of healthy children and patients are shown in Fig. 1.

Isolation and validation of serum exosomes. Exosomes were isolated from the serum of four healthy children and five adenovirus-infected patients. The patients were included in the study according to the criteria in Table IV. The isolated exosomes were characterized by TEM, and appeared as spherical vesicles of $30-100 \mathrm{~nm}$ in diameter in all samples (Fig. 2A), which is consistent with previously reported characteristics of exosomes $(20,22)$. In addition, exosome protein markers, namely CD9 and HSP90 $\alpha(22,23)$ were also detected in our exosome samples (Fig. 2B). CD9 and HSP90 $\alpha$ were highly expressed in the isolated exosomes compared with their levels in serum. These results suggest that exosomes were successfully isolated in the present study.

miRNA signatures of different exosomes. To compare the different miRNA signatures of patients with pneumonia caused by adenovirus infection with those of healthy volunteers, a total of 300 ng RNA isolated from $400 \mu$ l serum of each of the samples was used for miRNA-seq to compare the differentially expressed miRNAs in HAdV-infected patients. The expression of the miRNAs analyzed by miRNA-seq was compared between samples from four healthy controls and samples from five patients with HAdV infection (Fig. 3A). The majority of miRNAs exhibited significantly altered expression in $\mathrm{HAdV}$-infected patients, providing a clear distinction of HAdV pneumonia. Among the miRNAs evaluated, 18 were significantly upregulated while 10 were downregulated in patients compared with the normal controls (Fig. 3B). These results indicated that these miRNAs may serve as biomarkers for $\mathrm{HAdV}$-infected patients.

Pairwise RT-qPCR analysis of potential miRNA biomarkers for HAdV pneumonia. The present study further analyzed 
Table III. Primer sequence of nine miRs for quantitative polymerase chain reaction.

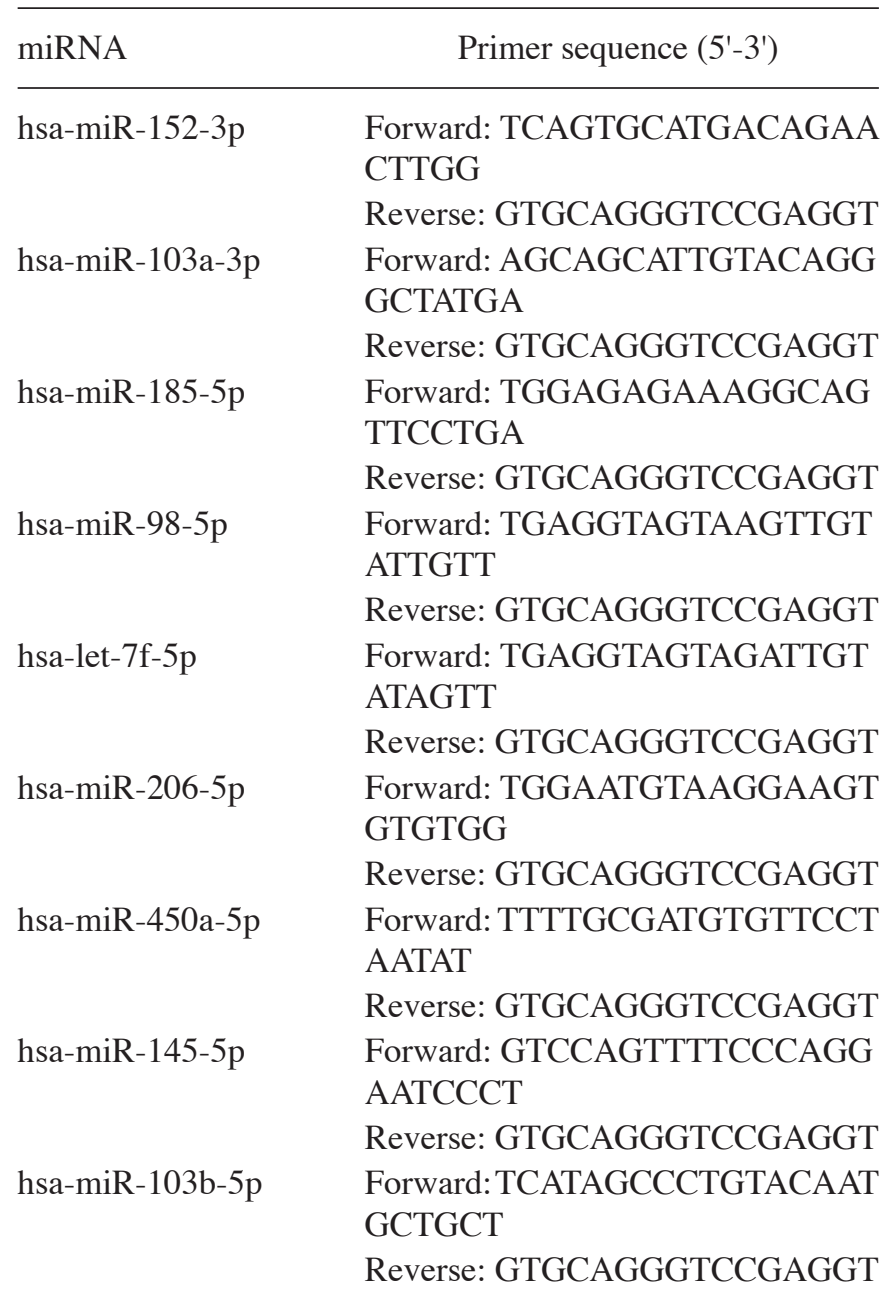

miR, microRNA.

the miRNAs with significantly different expression in HAdV-infected patients compared with healthy children. In total, the top nine differentially expressed miRNAs in order to analyze their association with pneumonia caused by HAdV infection. The expression of these nine miRNAs was evaluated in samples from five healthy children and five HAdV-infected patients. Importantly, an internal reference is essential in RT-qPCR experiments. However, in the case of miRNAs isolated from exosomes, an internal reference is not available. To solve this issue, pairwise analysis of each miRNA was used. It was observed that miR-103b-5p/miR-98-5p and miR-450a-5p/miR-103a-3p exhibited opposite expression trends. miR-103b-5p and miR-450a-5p were upregulated in HAdV samples while miR-98-5p and miR-103a-3p were downregulated in HAdV samples, indicating that this ratio may serve as a biomarker for HAdV pneumonia (Fig. 4). Notably, miR-103b-5p is a serum biomarker for colorectal adenocarcinoma (24). Thus, the present results suggested that the selected miRNAs may reflect HAdV infection.

Validation of potential biomarkers for HAdV pneumonia diagnosis. To verify the diagnostic capability of serum

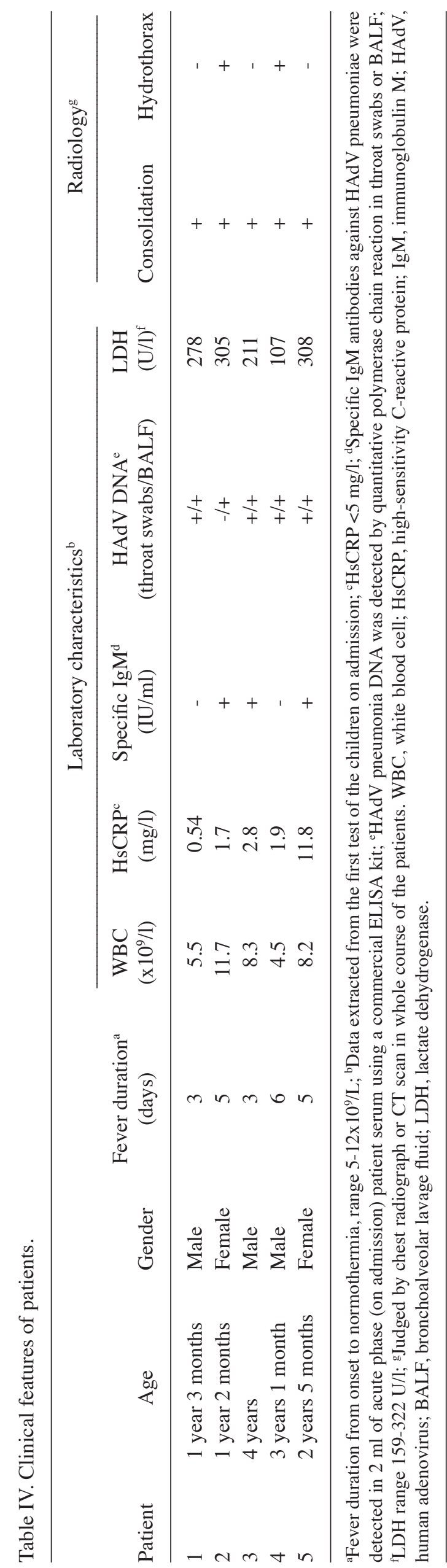


A
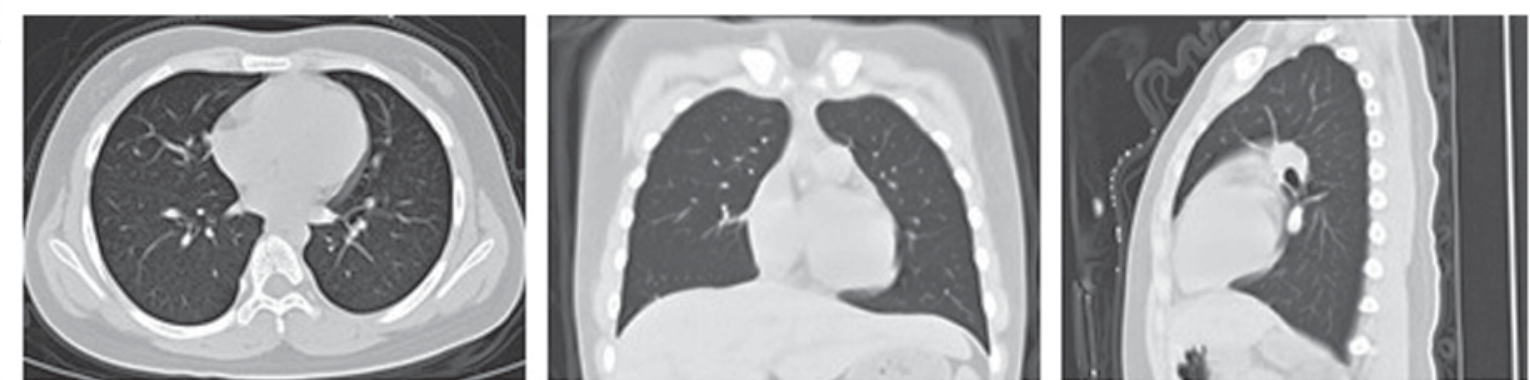

B
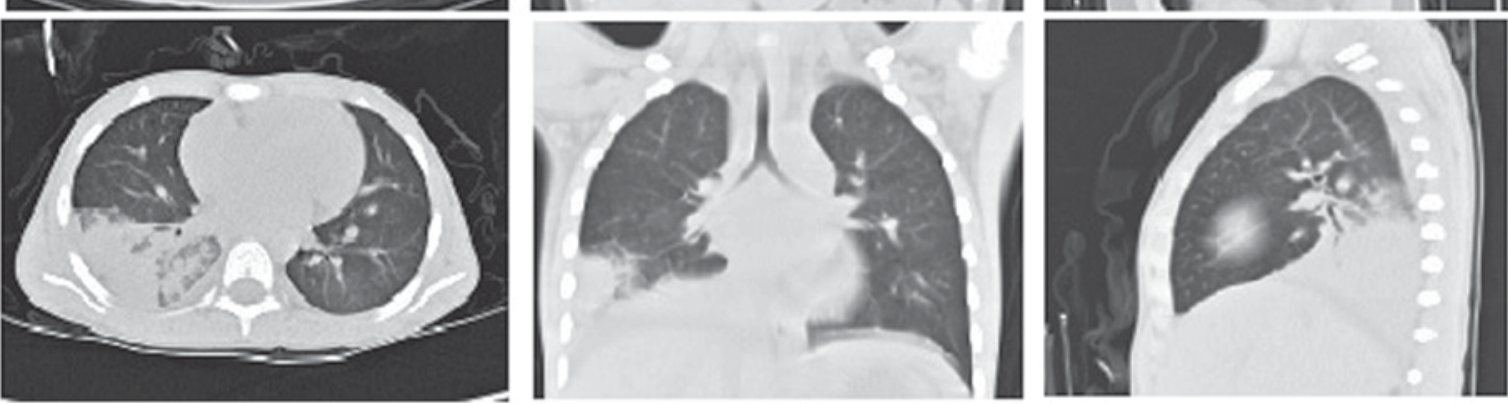

Figure 1. Chest radiograph of healthy children and HAdV-infected children. High-resolution computed tomography scan of the chest on the day of admission, revealing areas of airspace consolidation and pleural effusion in (A) healthy children and (B) patient 3 (4-year-old child with HAdV pneumonia). HAdv, human adenovirus.

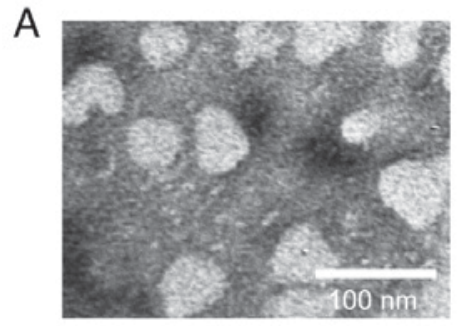

Control

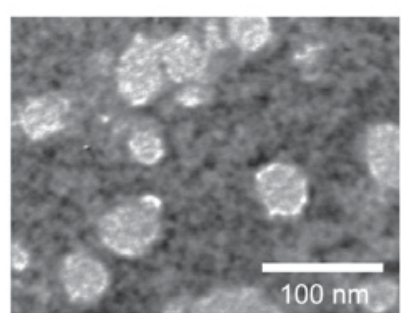

ADV

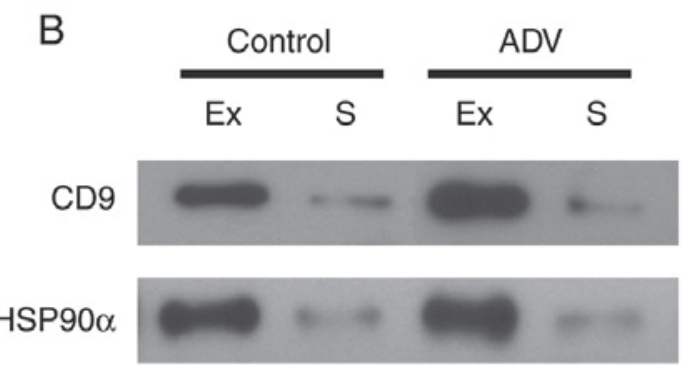

Figure 2. Characterization of isolated exosomes. (A) Transmission electron microscopy images of serum exosomes isolated from healthy controls (Control) and ADV patients. (B) The expression of exosome protein markers (CD9 and HSP90 $\alpha$ ) from the isolated exosomes was detected using western blot analysis. Equal quantities of protein were loaded in each lane. ADV, adenovirus-infected; Ex, exosome; S, serum; HSP90 $\alpha$, heat shock protein $90 \alpha$.

miRNAs as potential biomarkers in the present study, blood samples from 20 healthy children and $20 \mathrm{HAdV}$-infected patients were collected. The RT-qPCR results of the two pairs of miRNAs identified above were analyzed for all the samples in the validation cohort (Fig. 5). Relative $\mathrm{Cq}$ values for the miRNAs in the two pairs were subtracted from one another $[\mathrm{Cq}(\mathrm{miR}-450 \mathrm{a}-5 \mathrm{p})-\mathrm{Cq}(\mathrm{miR}-103 \mathrm{a}-3 \mathrm{p})$ and $\mathrm{Cq}(\mathrm{miR}-103 \mathrm{~b}-5 \mathrm{p})-\mathrm{Cq}(\mathrm{miR}-98-5 \mathrm{p})]$; subtracted values were used instead of ratios to simplify clinical analysis. The two pairs of miRNAs analyzed between healthy controls and HAdV patients were clearly separated as two clusters, suggesting that the miRNAs selected in the present study could be considered as good diagnostic biomarkers for HAdV pneumonia, at least in the cohort experiment. Importantly, neither pair of miRNAs could independently distinguish HAdV-infected patients from healthy children, highlighting the requirement for combining the two miRNA pairs identified in the present study.

\section{Discussion}

Serum miRNAs have been used as biomarkers for the diagnosis of various types of cancer (13). The associations between serum miRNAs and viruses have also been discussed 
A

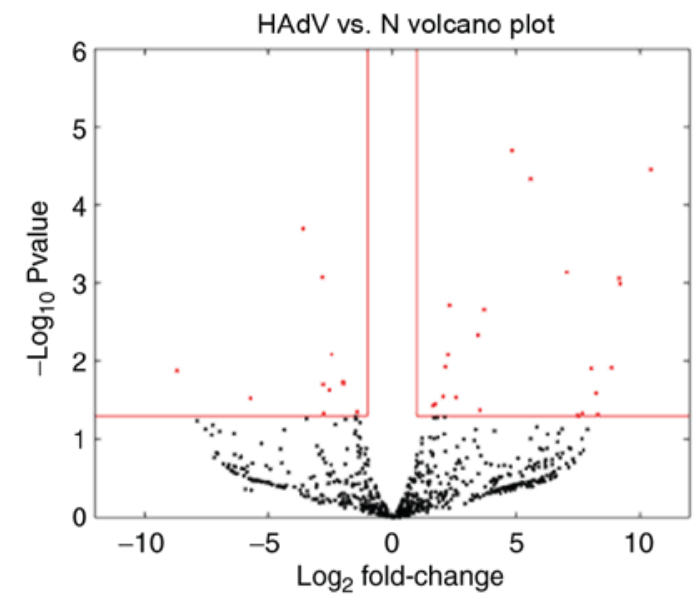

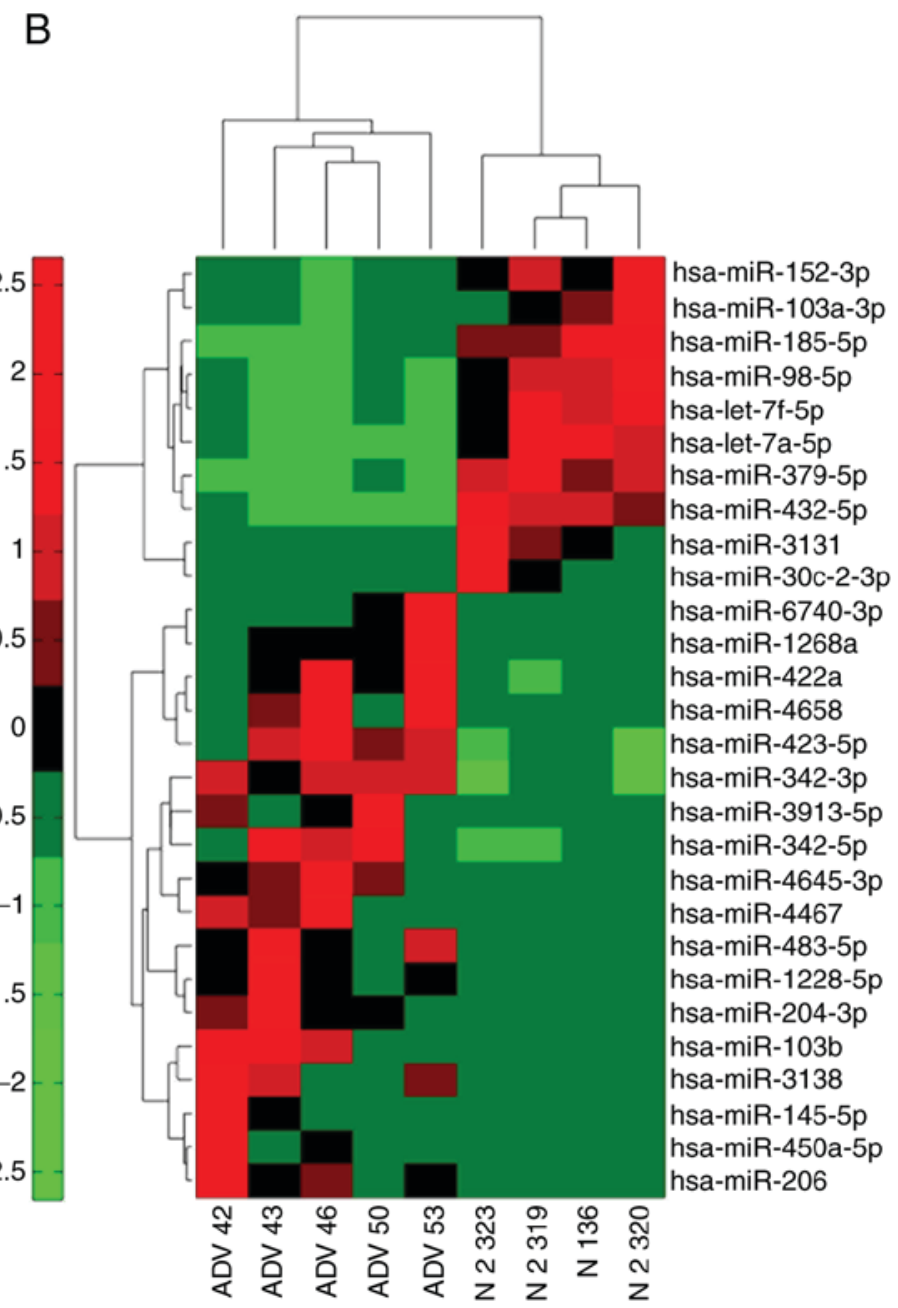

Figure 3. Differentially expressed miRs in the HAdV group compared with the control group. (A) Expression of exosomal miRs from the HAdV and control groups was evaluated by microarray analysis. (B) Heat map of miRs with differential expression in the HAdV or control groups. miR, microRNA; HAdv, human adenovirus; $\mathrm{N}$, healthy control.

in previous studies (25-27); however, there is no report thus far regarding serum miRNAs and pneumonia caused by $\mathrm{HAdV}$ infection. HAdVs are common pathogens that cause acute respiratory infections (1). The treatment of HAdV infections is controversial, as prospective, randomized therapeutic trials have not been conducted. Furthermore, severe HAdV pneumonia exhibits rapid development and a prolonged course in a proportion of patients with long-term respiratory problems, including bronchiectasis, bronchiolitis obliterans and hyperlucent lung $(28,29)$. In addition, an early diagnosis of pneumonia associated with HAdV infection is crucial but difficult. Therefore, it is important to identify biomarkers for HAdV pneumonia in children.

Recently, investigations of the function of miRNAs in lung diseases or those caused by viral infections have increased gradually (30-33). Furthermore, the roles of exosomal miRNAs have received increasing attention (34). Numerous studies have reported that changes in serum exosomes reflect different diseases and disease status $(35,36)$. Based on the available reports, miRNAs appear to be differentially enriched in serum exosomes. Additionally, the expression patterns of serum miRNAs change among different diseases. Therefore, exosomal miRNAs are considered potential diagnostic biomarkers for several diseases $(30,32)$. The use of exosomal miRNAs as biomarkers may be useful for the early diagnosis of diseases.

Our study screened HAdV-specific serum miRNAs. Although exosomal miRNAs were proposed as potential biomarkers for diseases several years ago, limited information has been identified and applied in clinical practice for the management of pneumonia in adenovirus-infected children to date. To the best of our knowledge, this is the first study to identify two pairs of exosomal miRNAs isolated from serum that can be considered as biomarkers for pneumonia in adenovirus-infected children. Using this approach, the present study identified that these two pairs of miRNAs were differentially expressed in the serum of HAdV-infected children compared with normal controls. However, additional samples should be evaluated in the future to identify other potential biomarkers. Our study has provided promising, non-invasive biomarkers for $\mathrm{HAdV}$ pneumonia.

The present study identified a unique expression profile for $\mathrm{HAdV}$ infection-associated serum miRNAs, namely miR-450a-5p/miR-103a-3p and miR-103b-5p/miR-98-5p. The results of our study agree with previously published reports 


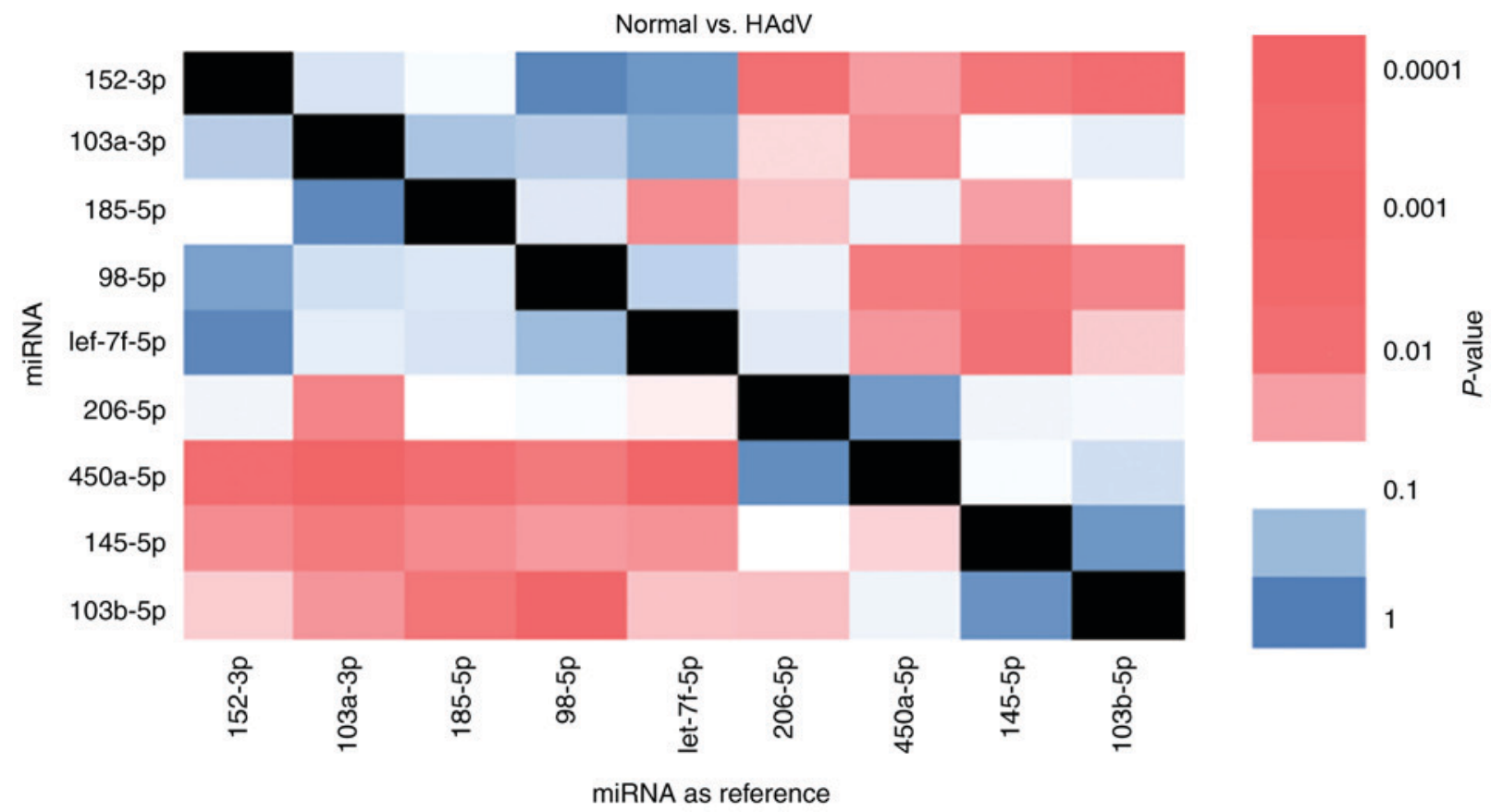

Figure 4. Correlation graph of the expression of nine miRNAs in the HAdV group compared with the control group using a pairwise t-test. A cohort of five healthy children and five HAdV-infected children was used to examine the expression levels of these nine candidate miRNAs via reverse transcriptionquantitative polymerase chain reaction analysis. The P-values of the pairwise t-tests between each pair of groups are shown in a color scale, where black indicates that the P-value could not be calculated. HAdv, human adenovirus; miRNA, microRNA.

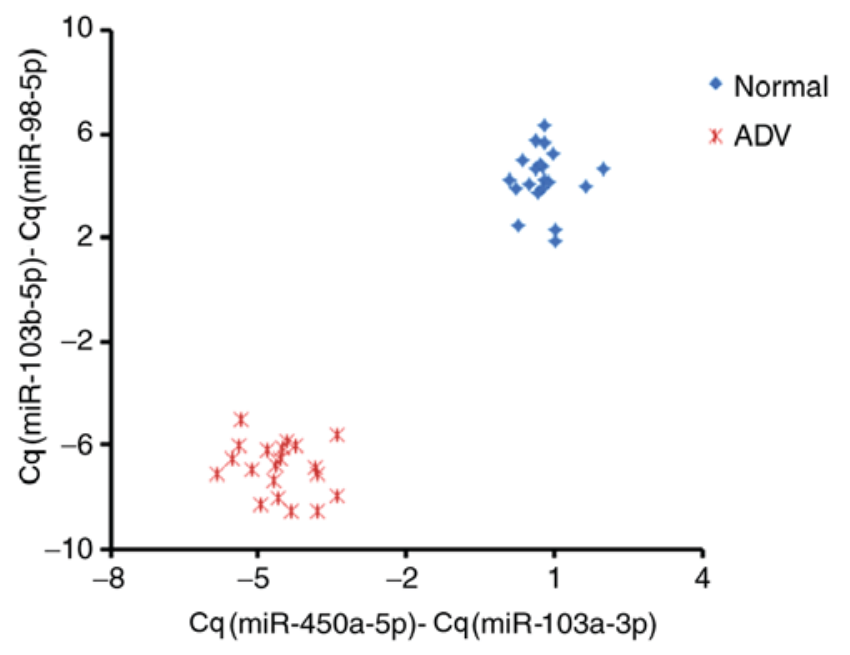

Figure 5. Validation of the potential biomarkers for adenovirus-infected pneumonia diagnosis. The $\mathrm{C}_{\mathrm{q}}$ values of the two pairs of candidate microRNA biomarkers are shown. Two-way analysis of variance revealed a significant interaction between patient status and miRNA pairs; $\mathrm{P}<0.01$. $\mathrm{C}_{\mathrm{q}}$, cycle quantification.

to some extent. In particular, miR-103b-5p has been reported as a serum biomarker for colorectal adenocarcinoma (24). miR-103a-3p was identified in serum, and was considered to serve an important role in lung adenocarcinoma (37). Serum miR-450a-5p has been reported to be a biomarker for several types of cancer (38); however, the function of serum miR-98-5p has not been discussed thus far. In addition to their potential role as biomarkers, miR-450a-5p was reported to control the mRNA expression of signal transducer and activator of transcription 1 (39) and miR-98-5p inhibited the activation of interferon (IFN)- $\alpha$ signaling (40), indicating that miR-450a-5p and miR-98-5p may serve roles in the IFN signaling pathway and may influence the progression of HAdV. Furthermore, serum miR-103a-3p is reported to be involved in the differentiation of Th2/Th17 cells in severe equine asthma (41), indicating that miR-103a-3p may affect the immune reaction in patients with $\mathrm{HAdV}$ infection. However, as a limited number of samples were included in the present study, additional samples should be evaluated to confirm the diagnostic capabilities of the aforementioned miRNAs for adenovirus infection-associated pneumonia, and further experiments should be performed to clarify the underlying mechanism in the future.

In conclusion, the expression profile of serum miRNAs may provide novel biomarkers for the diagnosis of pneumonia in adenovirus-infected children. miR-450a-5p/miR-103a-3p and $\mathrm{miR}-103 \mathrm{~b} / \mathrm{miR}-98-5 \mathrm{p}$ could be considered as potential diagnostic biomarkers for adenovirus infection-associated pneumonia.

\section{Acknowledgements}

Not applicable.

\section{Funding}

The present study was supported by the National Science and Technology Major Project (grant no. 2018ZX10101004003001), the National Natural Science Foundation of China (grant nos. 81601759 and 81701990), the Science and Technology Project of Guangdong Province, China (grant no. 2014A020212020) and the General Projects of Guangzhou Scientific Research (grant no. 201707010183). 


\section{Availability of data and materials}

The datasets used and/or analyzed during the current study are available from the corresponding author on reasonable request.

\section{Authors' contributions}

GL made significant contributions towards the design of the present study. DY and HF participated in sample diagnosis and collection. FH, JZ, JB and TS performed the experiments. LH and $\mathrm{HF}$ were responsible for the statistical analysis. FH, JZ and GL drafted, revised and edited the manuscript. All authors read and approved the final manuscript.

\section{Ethics approval and consent to participate}

The study was approved by the Ethics Committee at Guangzhou Women and Children's Medical Center (Guangzhou, China). All the parents or legal guardians of the patients signed written informed consent forms and agreed to its content.

\section{Patient consent for publication}

All the parents or legal guardians of the patients signed written informed consent forms and agreed to its content.

\section{Competing interests}

The authors declare that they have no competing interests.

\section{References}

1. Agweyu A, Kibore M, Digolo L, Kosgei C, Maina V, Mugane S, Muma S, Wachira J, Waiyego $M$ and Maleche-Obimbo E: Prevalence and correlates of treatment failure among Kenyan children hospitalised with severe community-acquired pneumonia: A prospective study of the clinical effectiveness of WHO pneumonia case management guidelines. Trop Med Int Health 19: 1310-1320, 2014.

2. Bhutta ZA, Das JK, Walker N, Rizvi A, Campbell H, Rudan I and Black RE; Lancet Diarrhoea and Pneumonia Interventions Study Group: Interventions to address deaths from childhood pneumonia and diarrhoea equitably: What works and at what cost? Lancet 381: 1417-1429, 2013.

3. Kim SJ, Kim K, Park SB, Hong DJ and Jhun BW: Outcomes of early administration of cidofovir in non-immunocompromised patients with severe adenovirus pneumonia. PLoS One 10: e0122642, 2015.

4. Nascimento-Carvalho CM: Etiology of childhood community acquired pneumonia and its implications for vaccination. Braz J Infect Dis 5: 87-97, 2001.

5. Elenius V, Peltola V, Ruuskanen $O$, Ylihärsilä $M$ and Waris $M$ : Plasma procalcitonin levels in children with adenovirus infection. Arch Dis Child 97: 582-583, 2012.

6. Girouard G, Garceau R, Thibault L, Bourque C, Bastien N and Li Y: Province-wide adenovirus type 3 outbreak with severe cases in New Brunswick. Can J Infect Dis Med Microbiol 22: e4-e6, 2011

7. Lynch JP III, Fishbein M and Echavarria M: Adenovirus. Semin Respir Crit Care Med 32: 494-511, 2011

8. Lynch JP III and Kajon AE: Adenovirus: Epidemiology, global spread of novel serotypes, and advances in treatment and prevention. Semin Respir Crit Care Med 37: 586-602, 2016.

9. Bartel DP: MicroRNAs: Genomics, biogenesis, mechanism, and function. Cell 116: 281-297, 2004.

10. Yan S, Han B, Gao S, Wang X, Wang Z, Wang F, Zhang J, Xu D and Sun B: Exosome-encapsulated microRNAs as circulating biomarkers for colorectal cancer. Oncotarget 8: 60149-60158, 2017.

11. Tanaka T, Tanaka M, Tanaka T and Ishigamori R: Biomarkers for Colorectal Cancer. Int J Mol Sci 11: 3209-3225, 2010.
12. Clancy C, Joyce MR and Kerin MJ: The use of circulating microRNAs as diagnostic biomarkers in colorectal cancer. Cancer Biomark 15: 103-113, 2015.

13. Keller A, Leidinger P, Gislefoss R, Haugen A, Langseth H, Staehler P, Lenhof HP and Meese E: Stable serum miRNA profiles as potential tool for non-invasive lung cancer diagnosis. RNA Biol 8: 506-516, 2011.

14. Geekiyanage H, Jicha GA, Nelson PT and Chan C: Blood serum miRNA: Non-invasive biomarkers for Alzheimer's disease. Exp Neurol 235: 491-496, 2012.

15. Gallo A, Tandon M, Alevizos I and Illei GG: The majority of microRNAs detectable in serum and saliva is concentrated in exosomes. PLoS One 7: e30679, 2012.

16. Rekker K, Saare M, Roost AM, Kubo AL, Zarovni N, Chiesi A, Salumets A and Peters M: Comparison of serum exosome isolation methods for microRNA profiling. Clin Biochem 47: 135-138, 2014.

17. Huang F, Zhang J, Zhang Y, Geng G, Liang J, Li Y, Chen J, Liu C and Zhang H: RNA helicase MOV10 functions as a co-factor of HIV-1 Rev to facilitate Rev/RRE-dependent nuclear export of viral mRNAs: Virology 486: 15-26, 2015.

18. Zhang J, Huang F, Tan L, Bai C, Chen B, Liu J, Liang J, Liu C, Zhang S, Lu G, et al: Host protein moloney leukemia virus 10 (MOV10) acts as a restriction factor of influenza a virus by inhibiting the nuclear import of the viral nucleoprotein. J Virol 90: 3966-3980, 2016.

19. Chaudhary K, Poirion OB, Lu L and Garmire LX: Deep learning-based multi-omics integration robustly predicts survival in liver cancer. Clin Cancer Res 24: 1248-1259, 2018.

20. Jia HL, Zeng XQ, Huang F, Liu YM, Gong BS, Zhang KZ, Zeng JH, Guo DG, Wang ZY and Li YG: Integrated microRNA and mRNA sequencing analysis of age-related changes to mouse thymic epithelial cells. IUBMB Life 70: 678-690, 2018.

21. Livak KJ and Schmittgen TD: Analysis of relative gene expression data using real-time quantitative PCR and the 2(-Delta Delta C(T)) method. Methods 25: 402-408, 2001.

22. Xie XF, Chu HJ, Xu YF, Hua L, Wang ZP, Huang P, Jia HL and Zhang L: Proteomics study of serum exosomes in Kawasaki disease patients with coronary artery aneurysms. Cardiol J, Apr 3, 2018 (Epub ahead of print).

23. Stamer WD, Hoffman EA, Luther JM, Hachey DL and Schey KL: Protein profile of exosomes from trabecular meshwork cells. J Proteomics 74: 796-804, 2011.

24. Zheng G, Wang H, Zhang X, Yang Y, Wang L, Du L, Li W, Li J, Qu A, Liu Y and Wang C: Identification and validation of reference genes for qPCR detection of serum microRNAs in colorectal adenocarcinoma patients. PLoS One 8: e83025, 2013.

25. Zhu Z, Qi Y, Ge A, Zhu Y, Xu K, Ji H, Shi Z, Cui L and Zhou M: Comprehensive characterization of serum microRNA profile in response to the emerging avian influenza A (H7N9) virus infection in humans. Viruses 6: 1525-1539, 2014

26. Zekri AN, Youssef AS, El-Desouky ED, Ahmed OS, Lotfy MM, Nassar AA and Bahnassey AA: Serum microRNA panels as potential biomarkers for early detection of hepatocellular carcinoma on top of HCV infection. Tumour Biol 37: 12273-12286, 2016.

27. Li LM, Hu ZB, Zhou ZX, Chen X, Liu FY, Zhang JF, Shen HB, Zhang $\mathrm{CY}$ and Zen K: Serum microRNA profiles serve as novel biomarkers for HBV infection and diagnosis of HBV-positive hepatocarcinoma. Cancer Res 70: 9798-9807, 2010.

28. Rudan I, Chan KY, Zhang JSF, Theodoratou E, Feng XL, Salomon JA, Lawn JE, Cousens S, Black RE, Guo Y, et al: Causes of deaths in children younger than 5 years in China in 2008. Lancet 375: 1083-1089, 2010.

29. Lee J, Choi EH and Lee HJ: Clinical severity of respiratory adenoviral infection by serotypes in Korean children over 17 consecutive years (1991-2007). J Clin Virol 49: 115-120, 2010.

30. Kishore A, Borucka J, Petrkova J and Petrek M: Novel insights into miRNA in lung and heart inflammatory diseases. Mediators Inflamm 2014: 259131, 2014

31. Hassan T, McKiernan PJ, McElvaney NG, Cryan SA and Greene CM: Therapeutic modulation of miRNA for the treatment of proinflammatory lung diseases. Expert Rev Anti Infect Ther 10: 359-368, 2012.

32. Shwetha S, Gouthamchandra K, Chandra M, Ravishankar B, Khaja MN and Das S: Circulating miRNA profile in HCV infected serum: Novel insight into pathogenesis. Sci Rep 3: 1555, 2013.

33. Bargalló ME, Guardo AC, Maleno MJ, Miralles L, Egaña-Gorroño L, Escribà T, García F, Gatell JM, Arnedo M and Plana M: Utility of systematic isolation of immune cell subsets from HIV-infected individuals for miRNA profiling. J Immunol Methods 442: 12-19, 2017. 
34. Liao J, Liu R, Yin LH and Pu YP: Expression profiling of exosomal miRNAs derived from human esophageal cancer cells by Solexa high-throughput sequencing. Int J Mol Sci 15: 15530-15551, 2014

35. Beltrami C, Clayton A, Phillips AO, Fraser DJ and Bowen T: Analysis of urinary microRNAs in chronic kidney disease. Biochem Soc Trans 40: 875-879, 2012.

36. Alipoor SD, Mortaz E, Garssen J, Movassaghi M, Mirsaeidi M and Adcock IM: Exosomes and exosomal miRNA in respiratory diseases. Mediators Inflamm 2016: 5628404, 2016.

37. Ito S, Kamoto Y, Sakai A, Sasai K, Hayashi T, Toyooka S and Katayama H: Unique circulating microRNAs in relation to EGFR mutation status in Japanese smoker male with lung adenocarcinoma. Oncotarget 8: 114685-114697, 2017.

38. Zhang Y, Yu M, Dai M, Chen C, Tang Q, Jing W, Wang H and Tian W: miR-450a-5p within rat adipose tissue exosome-like vesicles promotes adipogenic differentiation by targeting WISP 2 . J Cell Sci 130: 1158-1168, 2017.

39. Dernowsek JA, Pereira MC, Fornari TA, Macedo C, Assis AF, Donate PB, Bombonato-Prado KF, Passos-Bueno MR and Passos GA: Posttranscriptional interaction between miR-450a-5p and miR-28-5p and STAT1 mRNA triggers osteoblastic differentiation of human mesenchymal stem cells. J Cell Biochem 118: 4045-4062, 2017.
40. Dong G, Fan H, Yang Y, Zhao G, You M, Wang T and Hou Y: $17 \beta$-Estradiol enhances the activation of IFN- $\alpha$ signaling in $B$ cells by down-regulating the expression of let-7e-5p, miR-98-5p and miR-145a-5p that target IKKe. Biochim Biophys Acta 1852: 1585-1598, 2015.

41. Pacholewska A, Kraft M, Gerber V and Jagannathan V: Differential expression of serum MicroRNAs supports CD $4^{+}$ $\mathrm{T}$ cell differentiation into Th2/Th17 cells in severe equine asthma. Genes (Basel) 8: pii: E383, 2017.

This work is licensed under a Creative Commons Attribution-NonCommercial-NoDerivatives 4.0 International (CC BY-NC-ND 4.0) License. 